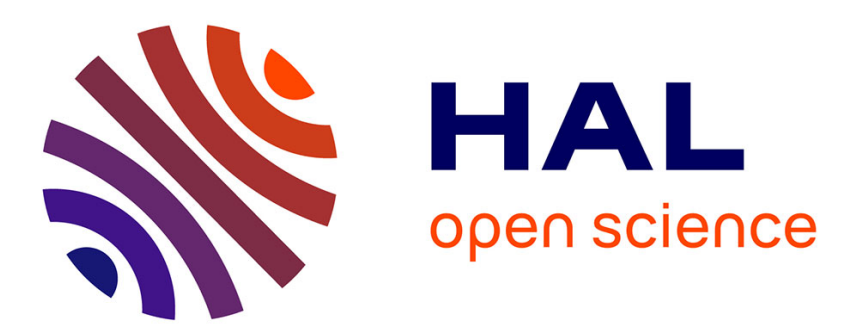

\title{
Validation d'un modèle tri-componentiel pour l'étude des représentations sociales à partir de mesures issues d'une tâche d'association verbale
}

Edith Salès-Wuillemin, Charles Galand, Sophia Cabello, Viviane Folcher

\section{- To cite this version:}

Edith Salès-Wuillemin, Charles Galand, Sophia Cabello, Viviane Folcher. Validation d'un modèle tri-componentiel pour l'étude des représentations sociales à partir de mesures issues d'une tâche d'association verbale. Les cahiers Internationaux de Psychologie Sociale, 2011, 91, pp.231-252. hal00645924

\author{
HAL Id: hal-00645924 \\ https://hal.science/hal-00645924
}

Submitted on 28 Nov 2011

HAL is a multi-disciplinary open access archive for the deposit and dissemination of scientific research documents, whether they are published or not. The documents may come from teaching and research institutions in France or abroad, or from public or private research centers.
L'archive ouverte pluridisciplinaire HAL, est destinée au dépôt et à la diffusion de documents scientifiques de niveau recherche, publiés ou non, émanant des établissements d'enseignement et de recherche français ou étrangers, des laboratoires publics ou privés. 


\section{Validation d'un modèle tri-componentiel pour l'étude des représentations sociales à partir de mesures issues d'une tâche d'association verbale \\ Validation of a tri-componential model for the study of the social representations built on measures stemming from a verbal association task}

Edith Salès-Wuillemin Edith.Sales-Wuillemin@univ-paris8.fr

Charles Galand* charles.galand@hotmail.fr Sophia Cabello* sophia.cabello@hotmail.fr

* Laboratoire Parisien de Psychologie Sociale (EA 4386)

Université Paris 8

2rue de la Liberté F-93526 Saint-Denis Cedex ** Laboratoire Paragraphe (EA 349)

Université Paris 8 2 rue de la Liberté F-93526 Saint-Denis Cedex

\section{Référence à mentionner pour citer cet article :}

Salès-Wuillemin, E., Galand, C., Cabello, S., Folcher, V. (2011). Validation d'un modèle tri-componentiel pour l'étude des représentations sociales à partir de mesures issues d'une tâche d'association verbale. Cahiers Internationaux de Psychologie Sociale, 91, 231-252. 


\section{Validation d'un modèle tri-componentiel pour l'étude des représentations sociales à partir de mesures issues d'une tâche d'associations verbales}

\section{Résumé :}

Les différents travaux portant sur la structure des représentations sociales (RS) ne s'accordent ni sur le nombre de dimensions organisatrices, ni sur la nature de leurs relations. L'objectif de cette étude est d'amorcer la validation d'un modèle tri-componentiel pour l'étude des RS. 437 étudiants en psychologie ont été sollicités pour répondre à une tâche daassociations verbales. Cette tâche comprenait une phase d'associations libres et une phase d'évaluation systématique des mots cités au moyen d'échelles. Une Analyse en Composantes Principales a fait apparaître trois axes indépendants et unipolaires correspondant aux trois dimensions : descriptive, fonctionnelle et évaluative, sous-tendues par le modèle. L'analyse a également permis d'identifier différentes catégories de contenus et de les hiérarchiser selon leur importance. Au-delà des enjeux liés à la validation d'un modèle tri-componentiel, cette étude met en relief l'intérêt de l'utilisation de questionnaires standardisés permettant un recueil stable des registres de connaissances impliqués dans les RS.

Mots clés : représentations sociales, approche structurale, modèle tri-componentiel, noyau central.

Abstract: Studies related to the structure of the social representations $(S R)$ neither agree on the number of organizing dimensions of the SR, nor on the nature of their relations. The objective of this study is to start validating a tri-componential model for the study of the SR. 437 students in psychology were solicited to answer a verbal association task. This task included 1/ a free association; and 2/ an evaluation of the induced words. The evaluation was made ûsing scales. An Analysis in Principal Components revealed three independent and unipolar axes corresponding to three dimensions: descriptive, functional and evaluative, underlined by the model. This analysis also permitted to identify various categories of contents and to treat them on a hierarchical basis according to their importance. Beyond the validation of a tri-componential model, this study highlights the interest of using standardized questionnaires measuring the different registers of knowledge implied in the SR. 
Key words: social representations, structural approach, tri-componential model, core of the social representation.

\section{Introduction}

Moscovici (1961) a initié un vaste champ de recherches en proposant la théorie des représentations sociales (RS). Depuis sa formulation initiale, la théorie a vu le développement de plusieurs courants parmi lesquels le structuralisme aixois suscite encore beaucoup d'intérêt (e.g. Abric, 1976, 1989, 1994, 2003 ; Flament, 1989, 1994, 2001 ; Rateau, 1999, 2000, 2002). Dans ce cadre, une RS est conçue comme un ensemble de connaissances doué de propriétés structurales. L'étude de ce savoir de «sens commun » revient alors à identifier les cognitions constitutives de la représentation, puis à dégager la structure élémentaire autour de laquelle le système s'articule. Dans la lignée de «l'Ecole d'Aix », de nombreux travaux ont tenté de décrire des dimensions qui concourent à l'organisation des représentations. Bien qu'il n'existe pour le moment pas de consensus sur la façon dont ces dimensions structurent le champ représentationnel, l'ensemble de ces recherches converge vers la mise en évidence de trois dimensions organisatrices. Cette étude vise à contribuer au développement d'une approche tricomponentielle pour l'étude des RS.

\section{$\underline{\text { La théorie du noyau central et la nature du champ représentationnel }}$}

Proposée par Abric (1976, 1987,1989,1993, 1994a, 1994b, 2003), la théorie du noyau central fédère les recherches sur les RS qui s'inscrivent dans une perspective structurale. Cette théorie postule que toute représentation s'organise autour d'un noyau central, système stable formé de quelques éléments donnant signification et cohérence à la représentation. Autour de ce noyau graviterait un plus grand nombre d'éléments périphériques dont l'activation, moins systématique et plus opérationnelle, permettrait de contextualiser le système central

Dans lâ continuité de cette théorie, d'autres travaux ont cherché à décrire la nature des cognitions constitutives de la représentation. Abric (1994a, 1994b) propose qu'une dimension fonctionnelle/normative parcoure le système central. Le noyau comprendrait trois types de cognitions : 1/ des éléments fonctionnels : constituant le cadre de référence des pratiques sociales et des pratiques opératoires, ces éléments organiseraient les conduites à adopter vis-àvis de l'objet en définissant les «pratiques légitimes » que les individus doivent mettre en place lorsqu'ils sont confrontés à l'objet ; $2 /$ des éléments normatifs : formant le cadre de 
référence à partir duquel l'objet est socialement évalué, ces éléments orienteraient les jugements et les prises de position à l'égard de l'objet en reflétant le système de valeurs du groupe social considéré ; 3/ des éléments mixtes : en intervenant autant dans la production de jugements que dans l'orientation des pratiques, ces éléments occuperaient un statut particulier au sein du système central en étant liés aux normes du groupe et aux pratiques sociales. Quelques résultats empiriques illustrent la répartition de la nature de ces cognitions. La recherche d'Abric et Tafani (1995), sur la RS de l'entreprise, révèle que la force d'activation et la nature fonctionnelle, normative ou mixte des éléments centraux dépendent de la relation que le groupe entretient avec l'objet. L'absence de pratiques aurait pour conséquence de saturer le système central d'éléments normatifs et de leur conférer une plus grande importance. Au contraire, l'expérience accumulée se traduirait par un statut plus important des éléments fonctionnels. Rateau (1995a) montre qu'une même RS peut être contextualisée soit dans un «système d'activité », soit dans un «système de normes ». En faisant varier le terme inducteur (groupe d'amis versus groupe d'amis idéal), la représentation du groupe « réel » active davantage de relations faisant référence à l'expérience quotidienne concrète, celle du groupe «idéalisé » active plutôt des relations faísant référence à des valeurs et à des jugements. L'auteur en conclue que «ces deux images s'organisent autour de deux principes organisateurs de natures différentes : l'une normative (liée aux valeurs, à l'idéologie ou à l'aspect socio-affectif de la représentation) et l'autre fonctionnelle (liée aux pratiques développées à l'égard de l'objet)»(p.36).

Flament (1994) suggère d'étendre la distinction des aspects fonctionnels et normatifs à l'ensemble des cognitions constitutives d'une RS. Il propose pour cela une dimension descriptive/prescriptive englobant celle avancée par Abric (op. cit.). Dans cette perspective, chaque élément, qu'il appartienne au système central ou au système périphérique, pourrait revêtir deux aspects : descriptif et/ou prescriptif. L'aspect descriptif serait le plus souvent approché, «en effet, les sujets, surtout dans les études de RS, utilisent principalement des termes descriptifs - qu'ensuite l'enquêteur reprend dans ses questionnaires systématiques » (Flament, op. cit., p.38). L'aspect prescripteur renverrait à la fois aux modalités d'exécution d'actions et aux règles qui y correspondent. Les aspects normatif et fonctionnel seraient les deux facettes d'une même prescription: «il nous semble nécessaire de rapprocher ce qu'Abric (1987, p.69) distingue en parlant d'une "dimension normative" et d'une "dimension fonctionnelle". Et alors, on comprend bien pourquoi Abric et Tafani (1995) trouvent 
expérimentalement que des éléments d'une représentation puissent être, à des degrés divers, à la fois fonctionnels et normatifs »(Flament, op. cit., p.38). De par leur double aspect, les prescriptions semblent occuper une place particulièrement importante (voir à ce sujet la théorie des prescriptions conditionnelles, Flament, 1987, 1994, 2001). Si la nature des cognitions contribue à la structure des RS, il convient alors d'intégrer ces dimensions organisatrices à la théorie du noyau central. Le modèle bi-dimensionnel a été formulé dans cette optique.

\section{$\underline{\text { Le modèle bidimensionnel }}$}

Ce modèle a été développé par Moliner (1992, 1993, 1994a, 1995, 1996)à parrtir d'une série d'expérimentations sur la RS de l'entreprise et du groupe idéal. Le modèle avance qu'en plus de la dimension centrale/périphérique, s'ajoute une secondedimension structurante: descriptivelévaluative, qui parcourait l'ensemble du champ représentationnel. Ces deux dimensions seraient indépendantes et bipolaires car elles opposeraient à leurs pôles respectifs les cognitions selon leur statut central versus périphérique, et leur orientation descriptive versus évaluative. Ces deux dimensions sont employées par Moliner pour définir quatre champs de cognitions propres aux RS :1/ le champ des normes: composé d'éléments centraux orientés vers le jugement, vers une activité d'évaluation, ces éléments seraient investis d'une valeur sociale homogène visant à saisir la qualité de l'objet de représentation ; 2/ le champ des définitions; constitué de cognitions centrales définitoires, ces éléments permettraient d'identifier les différentes formes d'apparition de l'objet et de mobiliser la représentation adéquate; 3 / le champ des attentes : regroupant les désirs et les craintes du groupe social à l'égard de l'objet, ces cognitions périphériques joueraient le rôle de critères d'évaluation attitudinale; 4/ le champ des descriptions : agencé en schéma opérationnel, ces cognitions périphériques faciliteraient le traitement d'information en supportant des processus de catégorisation.

Malgré l'intérêt indéniable que présentent les travaux de Moliner pour le développement d'une approche structurale des RS, le modèle bidimensionnel s'oppose aux dimensions précédemment mises en évidence à trois niveaux. 1/ La bipolarité de la dimension descriptivelévaluative « revient à supposer une corrélation négative forte entre les deux pôles. Ce qui est directement contraire à notre analyse considérant que "descriptif" et "prescriptif" (ou : "normatif") sont deux aspects sous lesquels un même schème peut se manifester » 
(Flament, 1994, p.38-39). L'hypothèse d'une dimension bipolaire s'oppose également à la «double nature » des éléments mixtes envisagés par Abric et Tafani (op. cit.). 2/ L'orthogonalité suggère que les dimensions du modèle sont indépendantes, en d'autres termes, que la nature descriptive ou évaluative d'une cognition n'a aucune influence sur son statut central ou périphérique. Or, les travaux de Guimelli et Rateau suggèrent l'inverse. «Certains éléments ont effectivement un statut central du fait de leur caractère hautement normatif. De même, au sein du système périphérique certains éléments apparaissent comme structuralement plus importants que d'autres de par leur nature normative » (Lheureúx, Rateau \& Guimelli, 2008, p.51). Si la normativité est effectivement un indicateur de l'importance des éléments constitutifs d'une RS, alors le principe d'ôrthogonalité des dimensions ne peut être soutenu. 3/ Les deux dimensions du modèle bidimensionnel n'ont pas le même pouvoir structurant. La première hiérarchise l'importance des éléments selon leur statut (central versus périphérique), la seconde indique, tout aú plus, la nature des cognitions (descriptive versus évaluative) sans prévaloir de leur importance au sein de la RS. Rizkallah (2003) rappelle qu'une dimension n'est structurante que si celle-ci détermine des relations entre éléments constitutifs d'une représentation. «En stipulant que "Profit" est plus évaluatif que "Hiérarchie", Moliner considère indirectement que l'élément "Profit" est en soi évaluatif de l'entreprise. Or, ceci n'est nullement structural parce que l'évaluativité et la descriptivité constituent des significations qu'un élément ne peut contenir en soi. C'est de la relation entre les éléments qu'émerge le sens »(Rizkallah, 2003, p.5). Si l'on souhaite mettre en évidence les dimensions organisatrices des RS, il convient alors de s'intéresser aux relations structurantes plutôt qu'à la nature des cognitions. Le modèle des Schèmes Cognitifs de Bases (SCB) s'inscrit directement dans cette optique.

\section{$\underline{\text { Une lecture tri-dimensionnelle du modèle des SCB }}$}

Proposế par 'Guimelli et Rouquette (Guimelli, 1990, 1993a, 1993b, 1994, 2003 ; Rouquette, 1990, 1994 ; Guimelli \& Rouquette, 1992), le modèle des SCB stipule qu'entre deux «éléments de connaissance» (mot ou expression qui opérationnalise la notion de 《cognème » définie par Codol, 1969), il existe une relation « $\mathrm{R}$ » formalisée par un connecteur « $\mathrm{Ci} »$. Le modèle propose d'investiguer la structure à partir des relations qu'entretiennent les éléments constitutifs d'une RS. En partant de l'hypothèse que les cognitions centrales sont dotées de fonctions : génératrice et organisatrice, il devient possible de repérer le statut des éléments selon leur niveau d'implication dans des relations 
structurantes. Le modèle compte 28 modalités que peut prendre un connecteur, celles-ci sont regroupées en 5 familles de schèmes : lexique, voisinage, composition, praxie, attribution. Ces familles expriment des relations très diverses : délimitation du champ lexical (lexique), inclusion dans des catégories ou des classes (voisinage et composition), modalités d'exécution d'actions (praxie), attribution de propriétés et inférence causale (attribution).

Une lecture dimensionnelle du modèle des SCB a été proposée par Rateau (1995b) (voir aussi : Rouquette \& Rateau, 1998). L'auteur a réalisé une méta-analyse sur 6 ensembles de corpus de données. Les résultats permettent d'observer une tripartition des connecteurs en trois «méta-schèmes ». 1/ Un méta-schème de description: constitué des trois schèmes : lexique, voisinage et composition, ce méta-schème serait activé lorsque le sujet est amené à décrire, à définir l'objet ou des situations qui y sont liées. Ce registre de connaissances correspondrait à la dimension descriptive des représentations, $2 \lambda$ Un méta-schème d'action : composé uniquement du schème praxie, l'activation de ce méta-schème mettrait en avant des acteurs, des outils et des moyens d'action en référence â des pratiques relatives à l'objet ou à des situations qui y sont liées. Ce registre de connaissances renverrait à la dimension fonctionnelle des représentations. 3/ Un méta-schème d'évaluation : représenté par le schème attribution, ce méta-schème s'activerait lorsque le sujet est amené à porter un jugement, à évaluer, à rattacher l'objet et les situations quí y sont liées à des normes ou à des valeurs. Ce registre de connaissances renverrait à la dimension évaluative des représentations. Rateau (ibid.) considère que ces trois méta-schèmes reflètent l'existence de trois dimensions structurantes qui parcourent l'ensemble du champ représentationnel. Le modèle tricomponentiel s'inscrit dans la continuité de ces travaux.

\section{Le modèle tri-componentiel}

Proposê à différentes reprises (Salès-Wuillemin, 2005 ; Galand, 2010a, 2010b), ce modèle défend l'idée que toute RS serait constituée de cognitions, plus ou moins partagées, entretenant entre-elles des relations sémantiques. A un niveau collectif, la prise en compte de ees relations permettrait de décrire la structure interne de la représentation. L'ensemble de ces relations s'inscrirait dans les trois registres de connaissances: descriptif, fonctionnel et évaluatif, décrits précédemment. Ces différents registres référeraient à trois dimensions indépendantes unipolaires traduisant la nature des liens entre éléments constitutifs d'une représentation. Ces trois dimensions seraient plus précisément des composantes permettant 
d'appréhender la dimension centrale/périphérique située en amont. Ce modèle suggère l'application d'un principe d'additivité pour le repérage du statut central ou périphérique. L'application de ce principe sous-tend que la centralité d'un élément est appréhendée à la fois quantitativement et qualitativement. D'un point de vue quantitatif, l'élément sera pré-senti comme central à mesure que sa capacité associative sera importante. D'un point de vue qualitatif, la polysémie d'une cognition centrale se manifestera par l'activation de plusieurs registres de connaissances.

Ce modèle permet d'envisager huit catégories d'éléments. La première catégorie correspond aux éléments «a-dimensionnels », qualifiés de «peu informatifs» en raison de leur faible implication dans des relations structurantes sur chacune des composantes du modèle. Les trois catégories suivantes correspondent aux éléments «unidimensionnels »de type : descriptif, fonctionnel et évaluatif. Ils occupent un statut d'éléments organisateurs mais ce statut se limite à un seul registre de connaissances. Ils sont impliquềs dans des relations structurantes liées : soit à la description, soit à la mise en œuvre de pratiques, soit à des jugements sur l'objet ou des situations qui y sont liées. Leur activation est conditionnée par celle du registre de connaissances associé. Les trois catégories suivantes regroupent les éléments mixtes «bidimensionnels » de type : descriptif/fonctionnel, descriptiflévaluatif, fonctionnel/évaluatif. Ils occupent un statut organisateur en structurant des relations issues de deux des trois registres de connaissances. Leur simple évocation active des schèmes de natures différentes : des scripts pour les éléments descriptifffonctionnel, des normes de jugement pour les éléments descriptiflévaluatif, des normes de comportement pour les éléments fonctionnel/évaluatif (voir à ce sujet : Dubois, 2002; Sénémaud \& Testé, 2007). La dernière catégorie est composée d'éléments mixtes «tridimensionnels ». Ils ont comme particularité d'être impliqués dans un grand nombre de relations issues des trois registres de connaissances, ce qui fait de ces éléments de véritables «concepts organisateurs ». En ce sens, le contenu de cette catégorie peut être qualifié de «très informatif » car il permet d'accéder à l'objet de représentation de faȩon transversale, quel que soit le registre de connaissances sollicité.

Quelques résultats empiriques confortent les idées défendues par le modèle tri-componentiel. Le principe d'additivité sous-tend que le potentiel organisateur d'un élément augmente à mesure qu'il occupe un rôle important sur chacune des dimensions. Les cognitions mixtes sont donc plus importantes dans l'organisation des représentations. Les expériences menées par Guimelli (1998a), sur les représentations du groupe idéal auprès d'étudiants et la 
représentation du délinquant auprès du personnel de police, soulignent que les cognitions centrales mixtes (à la fois normative et fonctionnelle) sont plus décisives dans la reconnaissance et l'identification de l'objet que les cognitions uniquement fonctionnelles. Les résultats obtenus par Guimelli et Rateau (2003), sur la représentation des études supérieures, mettent en avant une tendance similaire. Or, cette fonction de reconnaissance et d'identification de l'objet est généralement attribuée au système central (cf. Abric, 1989). Lees cognitions mixtes assureraient davantage cette fonction car elles permettent d'éviter des effets d'activation sélective, comme cela peut être le cas avec les éléments fonctionnels dónt l'activation semble dépendre à la fois du niveau de pratique à l'égard de l'objef et de la formulation de la consigne (cf. Rouquette \& Rateau, 1998). Par ailleurs, les différentes catégories de contenus envisagées dans le modèle tri-componentiel-ont été retrouvées par Rateau (2002) sur la représentation du travail. L'auteur a mis en évidence des cognitions « unidimensionnelles »: descriptives (intégration sociale, contraignant), fonctionnelles (accroissement des compétences, utilité sociale, indépendant, épanouissement), évaluatives (rémunération, satisfaction), mais aussi des cognitions mixtes «bidimensionnelles »: descriptive/fonctionnelle (adaptation), descriptive/évaluative (valorisant), fonctionnelle/évaluative (relations sociales), et une cognition mixte «tridimensionnelle » descriptive/fonctionnel/évaluatif (nécessité). Il reste à s'intéresser aux méthodes employées pour appréhender les dimensions strueturantes dans le cadre d'une approche tricomponentielle.

\section{La tâche d'associations verbates dans le cadre d'une approche tri-componentielle}

En tant que méthode exploratoire d'étude des RS, la tâche d'associations verbales permet de faire apparaître les dimensions latentes qui structurent l'univers sémantique et d'accéder aux noyaux figuratifs de la représentation étudiée (De Rosa, 1988). Cette tâche consiste à présenter un terme inducteur - il s'agit généralement de l'objet de représentation - et à demander au sujet de produire les mots ou expressions qui lui viennent spontanément à l'esprit. Plusieurs indicateurs sont utilisés pour identifier la nature des éléments recueillis.

La fréquence de citation indique le degré de partage des mots dans la population ciblée. Le rang d'évocation révèle la proximité de l'association à l'objet de représentation. Vergès $(1992,1994)$ propose de combiner ces deux indicateurs pour le repérage du noyau. Les éléments cités, en moyenne, plus fréquemment et dans les premiers rangs sont considérés comme potentiellement centraux. Toutefois, Abric (2003) rappelle que ce critère n'est pas un 
indicateur de centralité mais de prototypicalité. Le rang d'apparition est un indicateur de l'accessibilité en mémoire : «sur la base de résultats de la psychologie cognitive sur les temps de réaction verbale et sur la rapidité d'association, le rang d'évocation peut-être utilisé comme un indice de l'accessibilité prototypique »(De Rosa, 2003, p87). L'accessibilité des catégories sémantiques a été abondamment documentée en psychologie cognitive et en psycholinguistique, en particulier dans les travaux inscrits dans la théorie de la typicalifé (Rosch, 1973, 1975). Dans une tâche d'associations verbales, la fréquence de citation et le) rang d'évocation sont les deux critères d'évaluation de la saillance des mots. Cette saillance, comme indicateur de typicalité, a deux caractéristiques principales : 1/ une plus grande accessibilité : les éléments les plus typiques sont cités en premier ; $2 /$ une accessibilité partagée : les éléments les plus typiques sont cités par le plus grand nombre. Pour Dubois et Poitou (e.g. Dubois \& Poitou, 2002; Poitou, 2000 ; Poitou \& Dubois 1999), la saillance dépendrait: de données expérientielles au sens large, dés propriétés linguistiques de l'inducteur et de l'inventaire lexical disponible. La saillance des items lexicaux serait : «le reflet d'une expérience partagée par les différentes communautés interrogées, expérience à la fois pratique et quotidienne et discursive des lectures de matériaux linguistiques » (Dubois \& Poitou, 2002, p233-234). Ces conclusions rappellent la proposition de Nelson (1973) selon laquelle les catégories se développent à partir d'un noyau fonctionnel. Dans cette perspective, l'objet serait d'abord identifié à partin de ses propriétés expérimentées au moyen d'actions. Le rang d'évocation des mots les plus fréquemment cités permettrait donc d'approcher indirectement la composante fonctionnelle des représentations.

En ce qui concerne la composante descriptive, certaines tâches associatives s'appuient sur la caractérisation : capacité des éléments à identifier l'objet de représentation. Cette notion est employée pour aborder certaines propriétés structurales, notamment la connexité et l'appartenance au système central. La connexité est étudiée à partir d'un questionnaire de caractérisation ou méthode des choix par bloc (cf. Guimelli, 1989, 1998b ; Vergès, 2001 ; Abric, 1998, 2003) où le sujet est amené à regrouper les items les plus et les moins caractéristiques de l'objet. La caractérisation est aussi utilisée pour tester la centralité avec la technique de mise en cause (Moliner, 1988a, 1988b, 1998), la technique d'Induction par Scénario Ambigu (Moliner, 1993, 1994b) et la technique de reconnaissance de l'objet (Abric \& Vergès, 1994). Ces techniques s'appuient sur l'une des propriétés des éléments du noyau : faciliter la reconnaissance en étant caractéristique de l'objet de représentation. 
En ce qui concerne la composante évaluative, à l'exception du modèle des $\mathrm{SCB}$, les mesures relatives à cette dimension sont rarement employées dans les tâches d'associations verbales pour appréhender une quelconque propriété structurale. Ces mesures visent plutôt à approcher une composante attitudinale de la représentation. Un exemple est fourni par le « réseau d'associations » élaboré par De Rosa $(1995,2003)$. La tâche consiste à demander au sujet de construire, par le dessin, un réseau d'associations verbales à partir d'un terme inducteur (ou à partir d'une image, cf. De Rosa \& Farr, 2001). Le sujet indique la polarité respective des mots produits (positive, négative ou neutre). La valence peut être employée pour opposer des groupes de sujets selon leur appréciation positive ou négative de l'objet.

La mise en relation des composantes descriptive, fonctionnelle et évaluative est fondamentale dans la perspective d'une approche tri-componentielle des RS. Dans cette optique, les membres du LAPPS ${ }^{1}$ utilisent une tâche d'associations verbales dotée de trois échelles : rang d'évocation, caractérisation et valence, permettant d'appréhender les différentes composantes du modèle (Galand, 2007, 2010a, 2010b ; Karanafti, 2007 ; Gaillard, Urdapilleta \& Salès-Wuillemin, 2009 ; Galand \& Salès-Wuillemin 2009 ; Salès-Wuillemin, Morlot, Masse \& Kohler, 2009). Pourtant, aucune étude n'a cherché à vérifier que ces échelles décrivent bien les composantes du modèle tri-componentiel. Cette étude visait donc à amorcer la validation du modèle à partir de données issues de cette tâche d'associations verbales.

\section{Méthode}

437 étudiants $^{2}$ inscrits en Licence et Master1 de psychologie à l'université de Paris 8 ont été sollicités pendant les 30 dernières minutes de leur cours pour remplir une tâche d'association verbales avec comme inducteur : drogue. Cet inducteur a été choisi car il s'agit d'un objet d'étude «classique» dans le champ des RS (e.g. Echebarria Echabe \& coll., 1992; Katerelos, 2003; Dany \& Apostolidis, 2002, 2007 ; Dany, 2008). Quel que soit le positionnement par rapport à l'objet, il est relativement présumable de retrouver la notion de dépendance comme concept organisateur de la représentation. La passation était collective afin de placer les répondants dans les mêmes conditions de passation.

La tâche se déroulait en trois temps. Les sujets étaient d'abord amenés à énoncer les mots ou expressions (dix au maximum) qui leur venaient à l'esprit par rapport à l'inducteur : drogue.

\footnotetext{
${ }^{1}$ Laboratoire Parisien de Psychologie Sociale (EA 4386)

${ }^{2}$ Age moyen : 23,25 ans, écart-type : 7,35 ; répartition hommes/femmes : 13,33\%/86,67\%
} 
Une première mesure était fournie par le rang d'évocation des mots recueillis (de 1 à 10). La consigne était formulée ainsi : «L'évocation de LA DROGUE renvoie pour moi aux mots et expressions suivants. Je les inscrits dans l'ordre où ils me viennent à l'esprit ». Les sujets devaient ensuite évaluer le potentiel descriptif et évaluatif des mots évoqués. Ils disposaient pour cela de deux échelles de Likert. Une échelle de caractérisation (de 1 à 10) évaluait la capacité des termes produits à définir la drogue. La consigne était : «Je note de 1 à 10 les mots et expressions que j'ai écrits dans la première colonne en fonction de leur capacité à définir LA DROGUE ». Une échelle de valeur $(\mathrm{de}-3$ à +3$)$ visait à mesurer la connotation positive, négative ou neutre de ces mots par rapport à l'objet de représentation. La consigne était : « Je note de -3 à +3 les mots et expressions que j'ai écrits dans la première colonne en fonction de leur connotation positive ou négative par rapport à LA DROGUE ».

Cette étude ayant pour objectif de vérifier l'adéquation de l'outil au modèle tri-componentiel, les notes obtenues à l'échelle de valeur ont été recodées. Ces notes ont été mises en valeur absolue pour ne conserver que la force de la relation à l'inducteur sur cet aspect. Ainsi, conformément aux dimensions sous-tendues par le modèle, les trois échelles incluses dans l'analyse étaient unipolaires. Les traitements statistiques visaient à faire émerger les dimensions sous-tendues par le modèle à partir des données recueillies.

Ces traitements ont porté sur les mots cités par au moins $10 \%$ de sujets de l'échantillon. Ce seuil est régulièrement choisi pour la sélection de corpus issus de tâches d'associations verbales dans le champ des RS (cf. Salès-Wuillemin, 2005). Le test de la loi binomiale a permis de vérifier que ce seuil correspondait à une probabilité extrêmement faible que ces mots soient cités à une telle fréquence par hasard $(\mathrm{p}<.001)^{3}$. Le corpus sélectionné était composé de 10 motś : addiction, alcool, cannabis, cocaïne, danger, dépendance, hérö̈ne, maladie mort, seringue. Les observations comportant des non-réponses à l'une des deux échelles de caractérisation ou de valeur n'ont pas été prises en compte. Au final, 790 observations ont été retenues.

Les traitements statistiques ont consisté en la réalisation d'une analyse factorielle en composantes principales (ACP) sur les scores aux trois échelles pour l'ensemble des mots du

\footnotetext{
${ }^{3}$ Pour calculer la probabilité qu'un élément E soit cité apparaisse au sein du corpus : $\mathrm{p}_{\text {aléat }}(\mathrm{E})=($ maximum évocation $) / \mathrm{N}$ termes différents $=\mathrm{p} ; \mathrm{p}_{\text {alét }}(\mathrm{E})=10 / 870=0,011$ Probabilité qu'un élément $\mathrm{E}$ soit cité $\mathrm{n}$ fois par hasard pour $\mathrm{N}$ termes différents : $1-\mathrm{P}(\mathrm{n}, \mathrm{N}, \mathrm{p})=1-\left({ }_{\mathrm{N}}^{\mathrm{N}}\right) \mathrm{p}^{\mathrm{n}}(1-\mathrm{p})^{\mathrm{N}-\mathrm{n}}$ avec $\left({ }_{\mathrm{N}}^{\mathrm{N}}\right)=\mathrm{N}$ ! / $\mathrm{n} !(\mathrm{N}-\mathrm{n}) ! ; 1-\mathrm{P}(44,870,0,011)=6,88 \times 10^{-15}<.001$
} 
corpus sélectionné. L'examen des résultats a visé la description : 1/ des facteurs par le biais des niveaux de variance expliquée et du poids factoriel des variables sur les axes ; 2/ des observations par le biais d'une analyse comparative sur les résultats factoriels (Anova). Le test de signification employé dans l'analyse comparative ne visait qu'à détecter des tendances, il ne permet en aucun cas la généralisation des résultats à une quelconque population parente.

\section{Résultats}

Description des facteurs issus de l'analyse factorielle en composantes principales

L'ACP a été effectuée en appliquant une «rotation varimax normalisée ». Cette technique consiste à ajuster la représentation en faisant pivoter les axes par rapport à leurs origines. L'ensemble des facteurs a été retenu afin de permettre l'expression de toutes les configurations de résultats possibles. L'ACP a généré autant de facteurs qu'il y avait de variables incluses dans l'analyse. Le tableau ci-dessous indique la part de variance expliquée par chaque axe.

\section{Insérer ici tableau 1}

Toutes les composantes issues de l'ACP ayant été retenues, les trois facteurs représentent la totalité de la variance. La répartition de la part de variance expliquée étant parfaitement équilibrée (1/3 de la variance totale expliquée par chacun des axes), cela signifie que les trois dimensions jouent un rôle comparable. Ces résultats préconisent de retenir pour la suite de l'analyse un modèle tridimensionnel. L'examen des poids factoriels a permis de déterminer la nature des facteurs en observant la façon dont les variables se projettent sur les axes.

\section{Insérer ici tableau 2}

Quel que sojt l'axe, la contribution moyenne à la variance expliquée est de : 0,333. Ce critère semblait suffisamment discriminant pour repérer les variables qui contribuaient le plus à la variance de chaque axe. La valeur contribue davantage à la variance expliquée du premier axe ; il s'agit du rang pour le second, de la caractérisation pour le troisième. Ainsi, les variables qui contribuent le plus à la variance d'un axe renvoient systématiquement à l'une des trois mesures : rang, caractérisation, valeur. Au regard de la façon dont les variables se projettent sur les axes, il en est déduit que : 
- l'axe 1 est «l'axe de la valeur » car cette mesure est projetée sur le pôle positif de l'axe : plus la valeur est grande, plus le mot est connoté.

- l'axe 2 correspond à « l'axe de la primauté » car le rang est projeté sur le pôle négatif : plus le rang est bas, plus le mot est évoqué en premier.

- l'axe 3 est «l'axe inverse de la caractérisation » car cette mesure est projetée sur le pôle négatif: plus le score de caractérisation est élevé, plus le mot décrit l'objet de représentation.

La description de ces facteurs conforte l'idée d'un modèle tridimensionnel mettant en jeu trois dimensions indépendantes et unipolaires. La poursuite de l'analyse sur les observations a permis de distinguer les éléments selon la place qu'ils occupent au sein du modèle.

\section{Analyse comparative des scores moyens des éléments sur les trois axes}

Pour distinguer le rôle des éléments par rapport aux trois dimensions, une Anova a été effectuée à partir des résultats factoriels obtenus pour chacune des observations. La comparaison portait sur les scores moyens des mots sur les trois axes. La variable indépendante «type de mot » était catégorisée. Elle comprenait 10 modalités car 10 mots ont été retenus dans l'analyse (addiction, alco@l, cannabis, cocä̈ne, danger, dépendance, héroüne, maladie, mort, seringue). Les variables dépendantes correspondaient aux résultats factoriels des observations sur chacun des trois axes. Les trois variables dépendantes prises en compte étaient : «l'axe de la primauté », «l'axe inverse de la caractérisation », «l'axe de la valeur ». Les résultats de l'Anova ${ }^{4}$ ont permis d'observer un effet global de la variable catégorisée sur les trois variables dépendantes considérées simultanément (Lambda de Wilks $27 / 2272=0,68$, significatif au seuilp<.001) et prises séparément (pour «l'axe de la primauté »: $F_{9 / 780}=7,23$, $\mathrm{p}<.001$; pour $\ll$ laxe inverse de la caractérisation »: $F_{9 / 780}=10,56, \mathrm{p}<.001$; pour «l'axe de la valeur $\left.\gg, F_{9 / 780}=12,63, \mathrm{p}<.001\right)$. La présence de ces effets indique que les scores moyens observés varient selon le type de mot. La poursuite de l'analyse visait à identifier les mots poùr lesquels les variations observées sont les plus marquées.

Les comparaisons spécifiques opposaient systématiquement deux groupes : une modalité de la variable indépendante aux autres modalités (ce qui revient à comparer un mot à tous les

\footnotetext{
${ }^{4}$ Les tests de significativité servent à généraliser la présence d'un effet à la population parente. L'échantillon constitué ici n'est représentatif d'aucune population parente. Les conditions de validités des différents tests réalisés n'ont donc pas été vérifiées. Ces tests visaient uniquement à indiquer des tendances observées auprès des étudiants interrogés.
} 
autres). Etant donné qu'il y avait 10 mots, la famille de contrastes comprenait 10 comparaisons spécifiques. La non-orthogonalité des contrastes a conduit à opter pour le test de Sidàk approprié à ce type de comparaisons post hoc (cf. Chanquoy, 2005). Ce test consiste à ajuster le seuil $\alpha$ de signification du F selon l'inégalité de Sidàk ${ }^{5}$. Par conséquent, les différences significatives indiquées pour chaque contraste au seuil $\mathrm{p}<.10$ renvoient au seuil ajusté $\alpha^{\prime}=.01$. Le seuil choisi indique une tendance plutôt qu'un effet généralisable. Les résultats sont synthétisés dans le tableau ci-dessous.

\section{Insérer ici tableau 3}

Les différences significatives sur chaque axe permettent de repérer les positionnements les plus extrêmes au sein de l'analyse factorielle. Sur «l'axe de la primauté », le mot maladie est projeté sur le pôle négatif avec un score moyen significativement plus bas que les autres mots, tandis que les mots cannabis et dépendance sont projetés sur lelpôle positif avec un score moyen significativement plus élevé. Sachant que le pôle positif de cet axe correspond aux éléments cités dans les premiers rangs, il en est déduit que dépendance et cannabis jouent un rôle organisateur par rapport à la dimension fonctionnelle.

Sur «l'axe inverse de la caractérisation », les mots alcool, maladie et seringue sont projetés sur le pôle négatif avec un score moyen significativement plus bas que les autres mots, tandis que les mots cocaïne, héroïne et dépendance sont projetés sur le pôle positif avec un score moyen significativement plus élevé. Sachant que le pôle négatif de cet axe correspond aux éléments qui caractérisent le mieux la drogue, il en est déduit que cocaïne, héroüne et dépendance jouent un rôle important sur la dimension descriptive.

Sur «l'axe de la valeur éyaluative», les mots dépendance, mort et danger sont projetés sur le pôle négatif avec un score moyen significativement plus bas que les autres mots, tandis que les mots alcool et cannabis sont projetés sur le pôle positif avec un score moyen significativement plus élevé. Sachant que le pôle positif de cet axe correspond aux éléments qui sont le plus investis d'affects, il en est déduit que dépendance, mort et danger occupent une place importante sur la dimension évaluative.

Aucune différence significative n'a été mise en évidence pour le mot addiction.

\footnotetext{
${ }^{5}$ Soit $\alpha(\mathrm{EE})$ : l'erreur de première espèce pour l'ensemble des contrastes non-orthogonaux, et $\alpha(\mathrm{EC}):$ l'erreur de première espèce d'un contraste donné.

Pour assurer $\alpha(E E) \leq \alpha$, à partir de l'inégalité de Sidàk : $\alpha(E E) \leq 1-(1-\alpha(E C))^{1 / r}$, il faut tester chaque contraste au niveau de signification : $\alpha^{\prime}=1-(1-\alpha)^{1 / \mathrm{r}}$.

Pour $\alpha=.10$ et pour 10 comparaisons, la procédure de Sidàk donne $\alpha ’=.0104$.
} 
Ces résultats permettent de statuer sur le rôle des éléments au sein de l'analyse factorielle. Puisque les mots du corpus étudié occupent différentes places sur chacune des dimensions, il est possible de hiérarchiser leur importance. Le statut d'un élément confère à celui-ci des propriétés particulières pour rendre compte de l'objet de représentation. Ainsi, chaque dimension illustre un registre de connaissances par lequel l'élément est lié à l'objet. La hiérarchisation des éléments sur ces différents registres permet d'appréhender la dimension centrale/périphérique telle qu'elle est décrite dans le modèle tri-componentiel. Les principales tendances mises en évidence dans l'analyse comparative ont été synthétisées dăns le graphique ci-dessous afin d'appréhender cette dimension située en amont des trois composantes : descriptive, fonctionnelle et évaluative.

\section{Insérer ici figure 1}

La hiérarchisation des éléments du corpus au sein de la représentation fait apparaitre trois catégories d'éléments allant d'un niveau plus périphérique vers un niveau plus central.

La première catégorie regroupe les éléments qui semblent, à priori, ne pas occuper un statut particulier pour appréhender l'objet de représentation. Cette catégorie concerne les mots qui apparaissent : soit en retrait dans la représentation factorielle, c'est le cas du mot addiction, soit en contribuant à l'analyse en raison de fábles notes obtenues sur les différentes mesures, c'est le cas des mots : alcool, maladie et seringue.

La seconde catégorie rassemble les éléments qui permettent d'appréhender l'objet de représentation par le biais d'un registre spécifique de connaissances. Cette catégorie concerne les mots qui contribuent à $Y$ analyse factorielle en raison de scores plus élevés sur l'une des trois mesures. Ainsi, l'hérö̈ne et cocä̈ne sont les éléments les plus caractéristiques de la drogue et leur emploi relèverait plutôt d'un registre descriptif. Le cannabis rappelle plus rapidement l'objet et renverrait à un registre fonctionnel. Le danger et la mort sont davantage investis d'affects, leur utilisation par rapport à la drogue correspondrait à un registre évaluatif. La troisième catégorie est illustrée par le concept de dépendance qui rend compte de l'objet indépendamment du type de registre de connaissances activé. Cet élément contribue fortement à l'analyse factorielle par des valeurs importantes conjointement sur les trois mesures. Ce concept est rappelé en premier, il décrit et évalue l'objet de représentation, et occupe probablement une position centrale au sein de la représentation.

\section{Discussion}


L'analyse des relations entre dimensions organisatrices des RS à partir de données issues d'une tâche d'associations verbales apporte des retombées à trois niveaux.

A un niveau théorique, les résultats présentés confirment bien l'existence d'un modèle tridimensionnel. L'analyse factorielle a mis en évidence trois composantes : descriptive, fonctionnelle et évaluative, ayant comme propriétés d'être indépendantes et unipolaires. Une analyse spécifique sur les mots recueillis a révélé différentes catégories de contenus jouant des rôles distincts au sein du modèle. La dimension centrale/périphérique a pu être approchée par la hiérarchisation du statut des éléments sur les trois composantes.

A un niveau méthodologique, les résultats sont encourageants pour la construction d'un outil de recueil où les mesures concordent avec l'une des conceptions théoriques qui concoure à l'explication des relations entre l'objet de représentation et les mots produits lors d'une tâche d'associations verbales. Les résultats permettent d'amorcer la validation d'un outil de recueil adapté à une conception tri-componentielle des RS.

A un niveau empirique, les résultats indiquent que la drogue renvoie pour les étudiants interrogés à une dizaine de termes : addiction, alcool,cannabis, cocä̈ne, danger, dépendance, hérö̈ne, maladie, mort, seringue. Ces mots n'ont pas le même statut pour appréhender l'objet. Le cannabis active un registre fonctionnel, la cocä̈ne et l'hérö̈ne un registre descriptif, le danger et la mort un registre évaluatif. Si le cannabis renvoie effectivement à des pratiques sans caractériser la drogue et sans activer d'affects, alors il est probable que cette substance bénéficie d'un statut particulier et que ses conséquences négatives soient plus ou moins occultées. La dépendance semble l'unique élément capable d'activer les trois registres de connaissances. Si ce concept constitue effectivement le noyau de la RS, alors la prise en compte des croyances associées à la dépendance est capitale pour comprendre l'évaluation de la dangerosité des substances.

Bien que ces résultats soient avant tout exploratoires, il est nécessaire de souligner la valeur heuristique de la méthode employée. Celle-ci tient, en premier lieu, au contexte de réalisation de l'analyse dimensionnelle. L'analyse a été effectuée sur les termes les plus fréquemment associés à l'objet. Cela a pour effets : d'exclure les connaissances qui relèvent d'un niveau de représentation plus individuel, et de cibler l'analyse sur celles qui sont socialement partagées. La singularité de la méthode employée tient, en second lieu, à la diversité des mesures incluses dans l'analyse dimensionnelle: le rang d'évocation combiné à la fréquence de citation, les mesures de caractérisation et de valeur. La diversité de ces mesures offrait 
l'avantage de favoriser l'expression des diverses conceptions dimensionnelles largement documentées dans le champ des RS.

Si la méthode s'avère novatrice, il convient de préciser que l'analyse porte sur un niveau d'investigation très spécifique: un seul objet de représentation et une seule population. Bien que cela ait permis d'appréhender la dimension centrale/périphérique située en amont des trois composantes, le fait d'avoir centré les investigations empêche toute généralisation des résultats. Rien ne garantit de retrouver le même type de relations entre les dimensions constitutives des représentations dans un autre contexte. Pour être développées, ces conclusions nécessiteront des recueils complémentaires afin de consolider les résultats obtenus. Il ne peut être exclu que les résultats dépendent du mode de recueil et de la nature de l'objet. En ce qui concerne le mode de recueil, il est souhaitable de prendre à minima la précaution d'utiliser le même questionnaire afin de standardiser les conditions de passation. Si le questionnaire utilisé présente un intérêt indéniable il peut être considérablement amélioré en ajoutant une échelle supplémentaire mesưrant la composante fonctionnelle de façon explicite. En ce qui concerne la possible interférence de la nature de l'objet, il paraît nécessaire de multiplier les recueils en faisant varier des objets dont on peut à priori penser qu'il seront plus ou moins investis sur l'une des trois composantes du modèle. Cette étude n'est qu'une première étape dans la vahìdation d'un modèle tri-componentiel. Une analyse transversale sur un plus vaste ensemble de données semble également nécessaire pour une meilleure compréhension de l'organisation structurale et dimensionnelle des RS. 


\section{TABLEAUX ET FIGURES DU TEXTE}

$\underline{\text { Tableau } 1 \text { : Extraction des composantes principales }}$

\begin{tabular}{|c|c|c|c|}
\hline $\begin{array}{c}\text { facteurs \valeurs } \\
\text { propres }\end{array}$ & Valeur propre & $\begin{array}{c}\text { \% Total de } \\
\text { variance expliquée }\end{array}$ & $\begin{array}{c}\text { Cumul en \% de la } \\
\text { variance expliquée }\end{array}$ \\
\hline Axe 1 & 1,0000 & $33,33 \%$ & $33,33 \%$ \\
\hline Axe 2 & 1,0000 & $33,33 \%$ & $66,67 \%$ \\
\hline Axe 3 & 0,9999 & $33,33 \%$ & $100,00 \%$ \\
\hline
\end{tabular}

Tableau 2 : Poids factoriels des variables sur les trois axes de l'ACP

\begin{tabular}{|c|c|c|c|}
\hline Mesures & Axe 1 & Axe 2 & Axe 3 \\
\hline Rang & $-0,0158$ & $\underline{\mathbf{0 , 9 9 8 7}}$ & 0,0494 \\
\hline Caractérisation & 0,0791 & 0,0499 & $\underline{\mathbf{- 0 , 9 9 5 6}}$ \\
\hline Valeur & $\underline{\mathbf{0 , 9 9 6 8}}$ & 0,0159 & $-0,0789$ \\
\hline $\begin{array}{c}\text { Contribution moyenne à la variance expliquée } \\
\text { (calculée à partir des poids en valeur absolue) }\end{array}$ & $\mathbf{0 , 3 3 3 3}$ & $\mathbf{0 , 3 3 3 3}$ & $\mathbf{0 , 3 3 3 3}$ \\
\hline
\end{tabular}

Tableau 3 : Synthèse des différentes comparaisons spécifiques effectuées

\begin{tabular}{|c|c|c|c|}
\hline Mots & $\begin{array}{c}\text { Scores moyens } \\
\text { sur « l'axe de la } \\
\text { primauté » }\end{array}$ & $\begin{array}{c}\text { Scores moyens sur } \\
\text { «l'axe inverse de } \\
\text { la caractérisation » }\end{array}$ & $\begin{array}{c}\text { Scores moyens } \\
\text { sur « l'axe de la } \\
\text { valeur » }\end{array}$ \\
\hline Addiction & 0,108 & $-0,168$ & $-0,05$ \\
\hline Alcool & $-0,327$ & $\underline{\mathbf{0 , 6 3 9}}$ & $\underline{\mathbf{- 0 , 7 3 1}}$ \\
\hline Cannabis & $\underline{\mathbf{0 , 3 6 3}}$ & 0,226 & $\underline{\mathbf{0 , 6 2 4}}$ \\
\hline Cocaïne & $-0,053$ & $\underline{\mathbf{0 , 2 1 4}}$ & $-0,112$ \\
\hline Danger & 0,134 & $-0,086$ & $\underline{\mathbf{0 , 2 5 3}}$ \\
\hline Dépendance & $\underline{\mathbf{0 , 2 5 7}}$ & $\underline{\mathbf{0 , 3 4 4}}$ & $\underline{\mathbf{0 , 2 6 6}}$ \\
\hline Héroïne & $-0,259$ & $\underline{\mathbf{0 , 2 2 9}}$ & $-0,248$ \\
\hline Maladie & $\underline{\mathbf{0 , 5 7 9}}$ & $\underline{\mathbf{0 , 5}}$ & $-0,009$ \\
\hline Mort & $-0,343$ & 0,218 & $\underline{\mathbf{0 , 5 8}}$ \\
\hline Seringue & $-0,28$ & $\underline{\mathbf{0 , 5 5 8}}$ & $-0,224$ \\
\hline
\end{tabular}

Aide à la lecture des résultats :

Un score surligné en caractère gras indique que la comparaison spécifique est significative au seuil p<.10 (Test de Sidàk $F_{1 / 780}$ )

Sur l'axe de la primauté : un score négatif signifie que le mot considéré a plutôt été évoqué en dernier, un score positif signifie que ce mot a plutôt été évoqué en premier

Sur l'axe inverse de la caractérisation: un score négatif signifie que le mot considéré décrit plutôt bien la drogue, un score positif signifie que ce mot décrit plutôt peu la drogue

Sur l'axe de la valeur: un score négatif signifie que le mot considéré est plutôt peu connoté par rapport à la drogue, un score positif signifie que ce mot est plutôt connoté fortement 
$\underline{\text { Figure } 1 \text { : Statut des éléments et registre de connaissances }}$

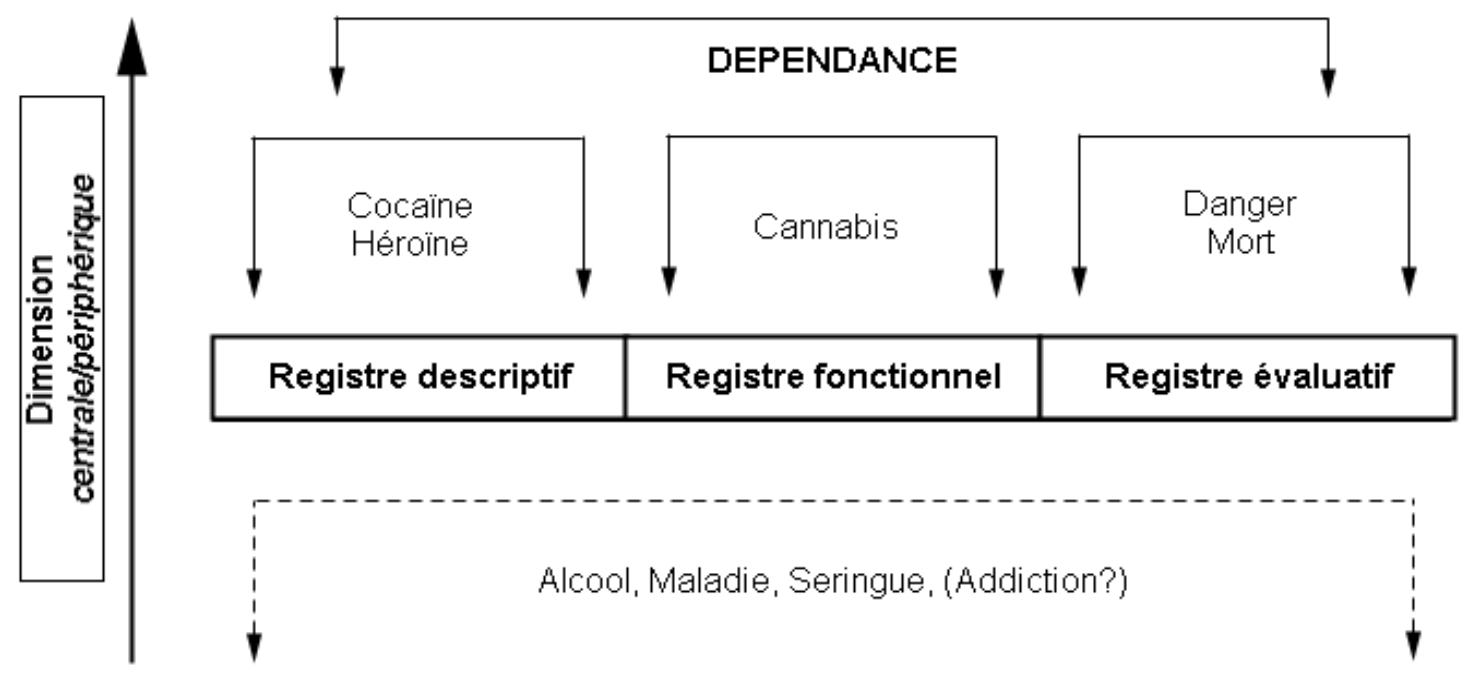




\section{REFERENCES}

Abric, J.-C. (1976). Jeux, conflits et représentations sociales. Thèse de doctorat, Université de Provence, Aix-en-Provence.

Abric, J-C. (1987). Coopération, compétition et représentations sociales. Cousset : Delval.

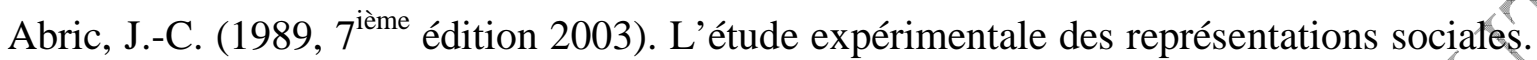
In D. Jodelet (Eds.), Les représentations sociales (pp.205-223). Paris : Presses Universitaires de) France.

Abric, J.-C. (1993). Central system, peripheral system: their functions and roles in the dynamics of social representations. Textes sur les représentations sociales-Papers on Social Representations, 2(2), 75-78.

http://www.psych.lse.ac.uk/psr/PSR1993/2_1993Abric.pdf

Abric, J-C. (Eds.) (1994, 4 ième édition 2003). Pratiques sociales et réprésentations. Paris : Presses Universitaires de France.

Abric, J-C. (1994a, 4 ième édition 2003). Les représentations sociales : aspects théoriques. In J.-C. Abric (Eds.), Pratiques sociales et représentations (pp.11-36). Paris : Presses Universitaires de France.

Abric, J.-C. (1994b). L'organisation interne des représentations sociales : système central, système périphérique. In C. Guimelli (Eds.), Structures et transformations des représentations sociales (pp.73-84). Neuchâtel, $\mathrm{CH}$ : Delachaux et Niestlé.

Abric, J.-C. (1998). L'étude des représentations sociales de la banque en France : une nouvelle approche méthodologique. In V. Rigas (Eds), Social representations and contemporary social problems (pp.2-10). Athène, GR: Ellinika Grammata.

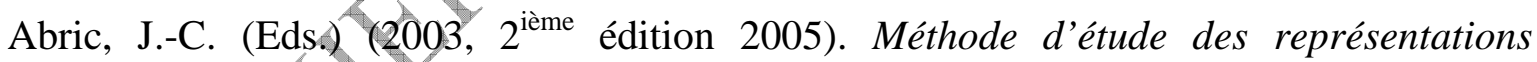
sociales. Ramonville Saint-Agne : Edition Erès.

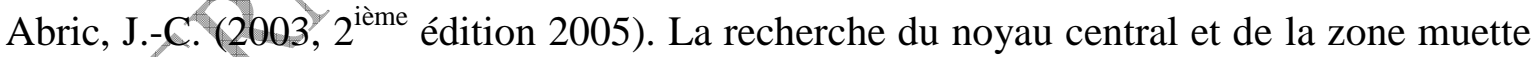
des représentations sóciales. In J.-C. Abric (Eds.), Méthode d'étude des représentations sociales (pp.59-80). Ramonville Saint-Agne : Erès.

Abric, J.-C., \& Tafani, C. (1995). Nature et fonctionnement du système central d'une représentation sociale: la représentation de l'entreprise. Les Cahiers Internationaux de Psychologie Sociale, 28(4), 22-31.

Abric, J.-C., \& Vergès, P. (1994). La représentation sociale de la banque. Etudes et recherches du Gifresh, n 26.

Chanquoy, L. (2005). Statistiques appliquées à la psychologie et aux sciences humaines et sociales. Paris : Hachette. 
Codol, J. P. (1969). Représentation de soi, d'autrui et de la tâche dans une situation sociale. Psychologie française, 14, 217-228. 134.

Dany, L. (2008). La drogue et le cannabis : approche psychosociale. Faire Savoirs, 7, 125-

Dany, L., \& Apostolidis, T. (2002). L'étude des représentations sociales de la drogue et du cannabis : un enjeu pour la prévention. Santé Publique, 14(4), 335-344

Dany, L., \& Apostolidis, T. (2007). Approche structurale de la représentation de la drogue : interrogations autour de la technique de mise en cause. Cahiers Internationaux de Psychologie Sociale, $73,11-26$.

De Rosa, A.S. (1988). Sur l'usage des associations libres dans l'étude des représentations sociales de la maladie mentale. Connexions, 51, 27-50.

De Rosa, A.S. (1995). Le «réseau d'associations » comme méthode d'étude dans la recherche sur les RS: structure, contenus et polarité du champ sémantique. Cahiers Internationaux de Psychologie Sociale, 1(28), 96-122.

De Rosa, A.S. (2003, $2^{\text {ième }}$ édition 2005). Le « réseau d'ássociations ». In J.-C. Abric (Eds.), Méthode d'étude des représentations sociales (pp.81-118). Ramonville Saint-Agne : Erès.

De Rosa, A.S., \& Farr, R.M. (2001). Icon and symbol. Two sides of the same coin in the investigation of social representations. In F. Buschini, \& N. Kalampalikis (Eds.), Penser la vie, le social, la nature. Mélanges en hommage ă Serge Moscovici (pp.237-256). Paris : Maison des Sciences de l'Homme.

Dubois, N. (2002). Autour de la norme sociale. Les Cahiers de Psychologie politique, 2 , Revue en ligne : http://lodel.irevues.inist.fr/cahierspsychologiepolitique/index.php?id=1640

Dubois, D., \& Poitou, J.(2002). Des « normes catégorielles »: structuration cognitive et/ou linguistique des catégories sémantiques. Intellectica, 2(35), 217-249.

Echebarria Echabe, A., Guede, E. F., Sanjuan Guillen, C., \& Valencia Garate, J.F. (1992). Social representations of drugs, causal judgment and social perception. European Journal of Social Psychology, 22(1), 73-84.

Flament,C. (1987). Pratiques et représentations sociales. In J.-L. Beauvois, R.-V. Joule, \& J.-M. Monteil (Eds.), Perspectives cognitives et conduites sociales, I, Théories implicites et conflits cognitifs (pp.143-150). Cousset : Delval.

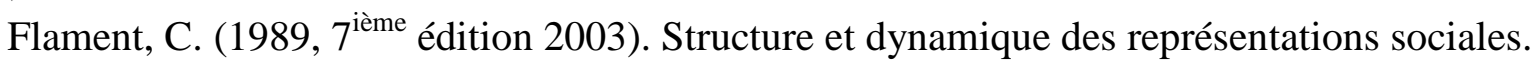
In D. Jodelet (Eds.), Les représentations sociales (pp.224-239). Paris : Presses Universitaires de France.

Flament, C. (1994, $4^{\text {ième }}$ édition 2003). Structure, dynamique et transformation des représentations sociales. In J.-C. Abric (Eds.), Pratiques sociales et représentations (pp.37-58). Paris : Presses Universitaires de France. 
Flament, C. (2001). Représentation sociale et normativité : quelques pistes. In F. Buschini, \& N. Kalampalikis (Eds.), Penser la vie, le social, la nature - Mélanges en l'honneur de Serge Moscovici (pp.257-261). Paris : Maison des Sciences de l'Homme.

Gaillard, A., Urdapilleta, I., \& Sales-Wuillemin, E. (2009). Représentation sociale de la nourriture chez des enfants de 6 à 11 ans. Communication et Psychologie Sociale, 22-24 juin, Tarbes.

Galand, C. (2007). De la genèse d'une représentation sociale à la perception des risques encourus - Appropriation différentielle des représentations sociales et perception des risques : application à l'usage de substances psychoactives. Mémoire de master II recherche, Université Paris VIII, Saint-Denis.

Galand, C. (2010a). Une approche tri-componentielle pour l'étude des représentations sociales - Développements théoriques et méthodologiques liés à la mise au point d'outils permettant d'appréhender le contenu et la structure d'une représentation. Thèse de doctorat, Université Paris VIII, Saint-Denis.

Galand, C. (2010b). Développements méthodologiques liés à Y'utilisation d'une tâche d'associations verbales pour appréhender le contenu et la structure d'une représentation sociale. Visioconférences internationales : Représentation Sociale et Culturelle, le 8 avril avec les Universités : Lyon 2, Paris 8, UQAM, Toulouse 2.

Galand, C., \& Salès-Wuillemin, E. (2009). La représentation des drogues chez les étudiants en psychologie : effets des pratiques de consommation et de l'entourage. Cahiers Internationaux de Psychologie Sociale, 84, 125-152.

Guimelli, C. (1989). Pratiques nouvelles et transformation sans rupture d'une représentation sociale : la représentation de la chasse et de la nature. In J.-L. Beauvois, R.-V. Joule, \& J.-M. Monteil (Eds.), Perspectives cognitives et conduites sociales, II, Représentations et processus socio-cognitifs (pp.117-138). Cousset : Delval.

Guimelli, C. (1990). Représentation sociale, noyau central et schèmes cognitifs de base. Nouvelles études psychologiques, 4(1), 27-44.

Guimelli, C. (1993a). Locating the central core of social representations: towards a method, European Journal of Social Psychology, 23(5), 555-559.

Guimelli, C. (1993b). Concerning the structure of social representations. Textes sur les représentations sociales - Papers on Social Representations, 2(2), 85-92.

http://www.psr.jku.at/PSR1993/2_1993Guime.pdf

Guimelli, C. (1994, $4^{\text {ième }}$ édition 2003). La fonction d'infirmière. Pratiques et représentations sociales. In J.-C. Abric (Eds.), Pratiques sociales et représentations (pp.83-108). Paris : Presses Universitaires de France.

Guimelli, C. (1998a). Differentiation between central core elements of social representations: normative vs. functional elements. Swiss Journal of Psychology, 57(4), 209224. 
Guimelli, C. (1998b). Chasse et Nature en Languedoc : étude de la dynamique d'une représentation sociale. Paris : L'Harmattan.

Guimelli, C. (2003, $2^{\text {ième }}$ édition 2005): Le modèle des schèmes cognitifs de base. Méthode et applications. In J.-C. Abric (Eds.), Méthode d'étude des représentations sociales (pp.119146). Ramonville Saint-Agne : Erès.

Guimelli, C., \& Rateau, P. (2003). Mise en évidence de la structure et du contenu d'une représentation sociale à partir du modèle des schèmes cognitifs de base (SCB) : la représentatíon des études. Nouvelle Revue de Psychologie Sociale, 2(2), 158-169.

Guimelli, C., \& Rouquette, M.-L. (1992). Contribution du modèle associatif des schèmes cognitifs de base à l'analyse structurale des représentations sociales. Bulletin de Psychologie, 405, 196-202.

Karanafti, A. (2007). La représentation des immigrés et de l'immigration chez les partisans du Front National en France selon leurs origines communautaires. Mémoire de master II recherche, Université Paris VIII, Saint-Denis.

Katerelos, I. (2003). Représentation sociale de la drogue chez les jeunes Grecs. Psychotropes, 9(1), 77-93.

http://www.cairn.info/load_pdf.php?ID_ARTICLE=PSYT_091_0077

Lheureux, F., Rateau, P., \& Guimelli, C. (2008). Hiérarchie structurale, conditionnalité et normativité des représentations sociales. Les Cahiers Internationaux de Psychologie Sociale, 77, 41-55.

Moliner, P. (1988a). La représentation sociale comme grille de lecture. Thèse de doctorat, Université de Provence, Aix-en-Provence.

Moliner, P. (1988b). Validation expérimentale de l'hypothèse du noyau central des représentations sociales. Bulletin de Psychologie, 387, 759-762.

Moliner, P. (1993). ISA : l'induction par scénario ambigu. Une méthode pour l'étude des représentations sociales. Revue Internationale de Psychologie Sociale, 6(2), 7-21.

Moliner, P. (1995). A two-dimensional model of social representations. European Journal of Social Psychology, 25(1), 27-40.

Moliner, P. (1996). Images et représentations sociales. De la théorie des représentations à l'étude des images sociales. Grenoble : Presse Universitaire de Grenoble.

Moliner, P. (1998). Dynamique et nature des représentations sociales. Cahiers Internationaux de Psychologie Sociale, 40, 62-70.

Moliner, P. (1992). La représentation sociale comme grille de lecture. Aix-en-Provence : Presses Universitaires de Provence.

Moliner, P. (1994a). Les deux dimensions des représentations sociales, Revue internationale de psychologie sociale, 73-86. 
Moliner, P. (1994b). Les méthodes de repérage et d'identification du noyau des représentations sociales. In C. Guimelli (Eds.), Structures et transformations des représentations sociales (pp.199-232). Neuchâtel, CH : Delachaux et Niestlé.

Moscovici, S. (1961, $3^{\text {̀̀me }}$ édition 2004). La psychanalyse son image et son public. Paris : Presses Universitaires de France.

Nelson, K. (1973). Structure and strategy in learning to talk. Monographs of the Society for Research in Child Developement, 38(149), 1-137.

Poitou, J. (2000). Prototypes, saillance et typicalité. Nouvelles terminologies, 21, 17-26.

Poitou, J., \& Dubois, D. (1999) Catégories sémantiques et cognitives : une étude expérimentale en sémantique lexicale, Cahiers de lexicologie, 74, 15-27.

Rateau, P. (1995a). Le noyau central des représentations sociales comme système hiérarchisé. Une étude sur la représentation du groupe. Les Cahiers Internationaux de Psychologie Sociale, 26, 29-52.

Rateau, P. (1995b). Dimensions descriptive, fonctionnelle et évaluative des représentations sociales. Une étude exploratoire. Papers on Social Representations, 4(2), 133-146.

Rateau P. (1999). Les effets d'un conflit d'idéntification idéologique sur la structure d'une représentation sociale. Les Cahiers Internationaux de Psychologie Sociale, 42, 90-101.

Rateau P. (2000). Idéologie, représentation sociale et attitude : étude expérimentale de leur hiérarchie. Revue Internationale de Psychologie Sociale, 13(1), 29-57.

Rateau, P. (2002). Procédure de substitution et nature des éléments des représentations sociales. Les Cahiers Internationaux de Psychologie Sociale, 54(2), 62-70.

Rizkallah E. (2003). Le modèle bi-dimensionnel des RS: Une critique théorique. Journal International sur les Représentations Sociales, 1(1), 1-10. http://geirso.uqam.ca/jirso/Vol1_Sept03/RizkallahE.pdf

Rosch, E. (1973). Natural Categories. Cognitive Psychology, 4, 328-350.

Rosch, E. (1975). Cognitive reference points. Cognitive Psychology, 7, 532-547.

Rouquette, M.-L. (1990). Sur la composition des schèmes. Nouvelles Etudes Psychologiques, 4(1), 17-25.

Rouquette, M.-L. (1994). Sur la connaissance des masses. Essai de psychologie politique. Grenoble : Presses Universitaires de Grenoble.

Rouquette, M-L., \& Rateau, P. (1998). Introduction à l'étude des représentations sociales. Grenoble : Presses Universitaires de Grenoble. 
Salès-Wuillemin, E. (2005). Psychologie sociale expérimentale de l'usage du langage Représentations sociales, catégorisation et attitudes: perspectives nouvelles. Paris : L'Harmattan.

Salès-Wuillemin, E., Morlot, R., Masse, L. Kohler, C. (2009) La représentation sociale de l'hygiène chez les professionnels de santé : Intérêt du recueil par entretien et de l'analyse discursive des opérateurs de liaison issus du modèle des Schèmes Cognitifs de Base (SCB). Cahiers Internationaux de Psychologie Sociale, 81, 43-72.

Sénémaud, C., \& Testé, B. (2007). La norme de consistance : une norme de jugement ou de comportement? Trente années de psychologie sociale avec J.-L. Beauvois: bilan \& perspectives, 27-29 juin, Université Paris V.

Vergès, P. (1992). L'évocation de l'argent: Une méthode pour la définition du noyau central d'une représentation. Bulletin de psychologie, 405, 203-209.

Vergès, P. (1994). Approche du noyau central : propriétés qualitatives et structurales, structures et transformations des représentations sociales. In C. Guimelli (Eds.), Structures et transformations des représentations sociales (pp.233-254). Neuchâtel, CH: Delachaux et Niestlé.

Vergès, P. (2001). L'analyse des représentations sociales par questionnaires. Revue de Sociologie Française, 42(3), 537-561. 